OPEN ACCESS

Edited by:

Jovan Pantelic,

University of California, Berkeley,

United States

Reviewed by:

Eric Teitelbaum,

Princeton University, United States

Shara Siby,

Indian Institute of Technology Madras,

India

*Correspondence:

Guillermo Rein

g.rein@imperial.ac.uk

Specialty section: This article was submitted to

Indoor Environment,

a section of the journal

Frontiers in Built Environment

Received: 30 November 2016

Accepted: 26 July 2018

Published: 22 August 2018

Citation:

Vermesi I, Restuccia F, Walker-Ravena C and Rein G (2018) Carbon Monoxide Diffusion Through

Porous Walls: Evidence Found in Incidents and Experimental Studies.

Front. Built Environ. 4:44. doi: 10.3389/fbuil.2018.00044

\section{Carbon Monoxide Diffusion Through Porous Walls: Evidence Found in Incidents and Experimental Studies}

\author{
Izabella Vermesi, Francesco Restuccia, Carlos Walker-Ravena and Guillermo Rein * \\ Department of Mechanical Engineering, Imperial College London, London, United Kingdom
}

It has been reported recently that carbon monoxide (CO) diffuses through gypsum board at a surprisingly high rate (Hampson et al., 2013). Because CO is poisonous and a by-product of systems typically present in residential housing such as boilers, generators and automobile engines, this finding could have a significant impact on the safety standards published by the National Fire Protection Association (NFPA) and International Code Council (ICC). In the USA, state legislation mandates the requirements for $\mathrm{CO}$ detection and warning equipment to be installed, but currently only enforces $\mathrm{CO}$ detection if there are communicating openings between the garage and occupied areas of a building. Therefore, there is a need to find out whether $\mathrm{CO}$ indeed diffuses through porous walls. In addition to investigating the validity of the experiments by Hampson (Hampson et al., 2013), this paper also collects a series of instances in the literature that show the diffusion of $\mathrm{CO}$ or other carbon-based gases. We have found a number of actual incidents and laboratory experiments which confirmed the transport of $\mathrm{CO}$ through other types of porous walls. We also found studies on the transport of other hydrocarbon gases with larger molecules than CO that can also diffuse through porous walls. We have also analyzed in detail the data from the recent experiments with a mass transfer model and confirm the validity of the findings for gypsum board. After $200 \mathrm{~min}$, the $\mathrm{CO}$ concentration in the control chamber was around 200 ppm, which is high enough to affect people. Our analysis independently confirms that $\mathrm{CO}$ can diffuse through porous walls at a fast rate and that the phenomena merits further research for consideration in life safety standards.

Keywords: carbon monoxide, diffusion, gypsum wallboard, carbon monoxide poisoning, mass transfer

\section{INTRODUCTION}

Carbon monoxide (CO) is a gas formed by the incomplete combustion of fuels such as wood, propane, gasoline, charcoal, natural gas and oil. It poses a threat to life safety, as it is poisonous in high concentrations due to its interference with oxygen transport in the respiratory system (Nelson, 1998). It is hard to detect as it is colorless and odorless.

Previously, it was thought that the threat of CO poisoning was confined to direct sources, such as gas cookers and coal-burning fires, and that if none of these sources were present inside a dwelling then the threat of $\mathrm{CO}$ intoxication was mitigated. However, this notion has now come under scrutiny due to investigation and reporting of several incidents in which $\mathrm{CO}$ might have been introduced into homes through non-communicating (i.e., no direct passage of air such as door or windows) walls and floors (Keshishian et al., 2012). 
The main driver of this investigation is a report (Hampson et al., 2013), in which CO is observed to transport from one chamber to an adjacent chamber by crossing a sample of gypsum wallboard. The aim was to study how fast a noxious concentration $(100 \mathrm{ppm})$ reaches the side of the gypsum board that has no source of CO. The gypsum boards used for this investigation were single layer $0.25^{\prime \prime}$ and $0.5^{\prime \prime}$ gypsum wallboards, as well as double layer $0.5^{\prime \prime}$ wallboard and double layer $0.5^{\prime \prime}$ wallboard that was painted on one side. For these wall configurations, the toxic concentration was reached in a much shorter time than was expected, i.e., from 17 to $96 \mathrm{~min}$, depending on the test. This was the first time the diffusion of CO through porous walls was studied.

The consequence of these findings is the acknowledgment of the increased susceptibility to $\mathrm{CO}$ intoxication and the possible changes in life safety legislation to accommodate for this previously dismissed pathway. The main standard that regulates the implementation of CO detection in the US is the NFPA 720, Standard for the Installation Of CO detection and Warning Equipment (NFPA, 2012), with the NFPA 101 Life Safety Code (NFPA, 2015) providing more general information related to this subject. For most types of buildings, such as hotels, dormitories, apartment buildings, lodging and day cares, these codes require the installation of $\mathrm{CO}$ detection only in dwellings that have fuel-fired equipment, fireplaces or which have a communicating attached garage. In the case of a non-communicating garage, there is no specific requirement because of the assumption that $\mathrm{CO}$ cannot travel through porous walls. However, the realization that this assumption is not valid and openings are not the only means of $\mathrm{CO}$ transmission may bring about stringent regulations regarding $\mathrm{CO}$ detection.

Such repercussions require the study (Hampson et al., 2013) and phenomenon to be independently confirmed. Therefore the purpose of this work is to further investigate whether $\mathrm{CO}$ can diffuse through porous walls. In order to do that, we produced a literature review to assess any previous studies that are relevant to the transport of $\mathrm{CO}$ through porous walls. Works focusing on the diffusion of gaseous species through membranes are reviewed. Afterwards, a mass transfer study of the experimental paper by Hampson et al. (2013) is performed using a simple mathematical model. The aim of the model is to verify if the results obtained in the experimental paper are reasonable and to check whether the phenomena merits further research.

\section{LITERATURE REVIEW}

\section{Diffusion in Porous Media}

Diffusion is the transport of mass from a region of higher concentration to a region of lower concentration (Incropera et al., 2013). There are several mechanisms of diffusion depending on the ratio between the mean free path of gas molecules and the mean pore diameter (Gilliland et al., 1974). Affinity toward transition (Knudsen) diffusion is seen when the mean free path of the molecules is larger than the mean pore radius of the porous medium, while a tendency for laminar/molecular flow is seen when the mean free path of the molecules is smaller than the mean pore radius of the porous medium. Essentially, this allows the characterization of these two diffusion mechanisms by their collisions: Knudsen diffusion constitutes moleculewall collisions and is typical of smaller pores whilst molecular diffusion is represented by molecule-molecule collisions and occurs in large pores (Kontogeorgos and Founti, 2013). A third diffusion mechanism has been observed in which the gas moves along the surface of the separating media, this form of diffusion is known as surface diffusion. The surface diffusion is typically of the order of $10^{-7}-10^{-9} \mathrm{~m}^{2} / \mathrm{s}$ (Treybal, 1981) which is several orders of magnitude smaller than both molecular and Knudsen diffusion.

The needle-like structure of the gypsum wallboard allows diffusion transport to occur due to a very complex process that involves molecular, Knudsen and surface diffusion within the porous interstices (Kontogeorgos and Founti, 2013). Various indoor climate experimental tests (Meininghaus et al., 2000; Meininghaus and Uhde, 2002; Blondeau et al., 2003), have studied the diffusion through porous walls of volatile organic compounds (VOC). The results presented in these works clearly show the transport of gases through the pores of the material. Therefore, the claim that $\mathrm{CO}$ diffuses through porous walls is supported.

\section{Experimental Studies Involving Drywall}

The effects of heating and air conditioning, interior doors, windows and exhaust fans on gas movement were evaluated using CO as the tracer gas in Chang and Guo (1992). The tests were carried out in a test house designed to replicate the interior of a residential dwelling. One of the test cases had the $\mathrm{CO}$ source in the bathroom, with the bathroom doors closed, the heating, ventilation and air-conditioning system of the house turned off, and the bathroom fan turned off. While the bathroom door was not purposely sealed off with impermeable materials, so some leakage might have existed, the main increase of $\mathrm{CO}$ concentration in the rest of the house was attributed to diffusion. The CO started to diffuse from the source room after the $10 \mathrm{~h}$, with the rate of diffusion increasing as the time passed.

The main transport process investigated in Singer et al. (2004) was sorption. As diffusion is a process that contributes to sorption it is of interest to relate the findings of this investigation. In this experiment a $50 \mathrm{~m}^{3}$ chamber with walls made from gypsum wallboard with a layer of low VOC flat latex paint was sealed. Twenty VOC gases were infused in the chamber, which was placed inside a test house. The gases were observed to diffuse through the gypsum walls of the chamber. The time frame for these experiments ranged from 2 to $12 \mathrm{~h}$. It was acknowledged that the infiltration rates to the chamber might reflect pore diffusion rates rather than air exchange.

Through the experimental study of indoor air quality, these two investigations confirm the possibility of $\mathrm{CO}$ transport through porous walls at a rate that presents a danger to people, despite the fact that the first case did not contain an airtight chamber, and the second investigated VOC. The first one represents a scenario that can be found in everyday conditions; therefore it is important to acknowledge the influence of diffusion. 
Another experiment that studies diffusion through gypsum walls was run by NIST researchers (Cleary et al., 2014) as a consequence of the study by Hampson et al. They measured the effective diffusion coefficient in an single-chamber experimental apparatus and found that the values they obtained matched the effective diffusion coefficients obtained in the reference work by Hampson et al. (for a board of $0.5^{\prime \prime}$ thickness, approximately $4.7 \times 10^{-6} \mathrm{~m}^{2} / \mathrm{s}$ for unpainted, $1.2 \times 10^{-6}$ $\mathrm{m}^{2} / \mathrm{s}$ for painted board), confirming the validity of the initial results. Additionally, Cleary et al. performed a simulation of CO transport through diffusion in a residential configuration and found that a concentration of $100 \mathrm{ppm}$ could be reached in less than $1 \mathrm{~h}$ for unpainted walls and less than $2 \mathrm{~h}$ for painted walls.

\section{Diffusion of Hydrocarbons Through Porous Walls}

Amongst the literature reviewed there were examples of other, larger hydrocarbon gases transported through porous interfaces. In particular, cases were found where gypsum wallboard was used.

Formaldehyde $\left(\mathrm{CH}_{2} \mathrm{O}\right)$ was used as the test gas in Deng et al. (2009). Four building materials were tested, namely particleboard, vinyl floor, medium-density board and highdensity board. Formaldehyde was observed to travel across them. Each of the four building materials' diffusion coefficient was evaluated at different temperatures: particleboard had the highest diffusivity $\left(3.18 \times 10^{-12} \mathrm{~m}^{2} / \mathrm{s}\right.$ at $\left.18^{\circ} \mathrm{C}\right)$ followed by high-density board $\left(6.87 \times 10^{-13} \mathrm{~m}^{2} / \mathrm{s}\right.$ at $\left.18^{\circ} \mathrm{C}\right)$, medium-density board $\left(7.68 \times 10^{-13} \mathrm{~m}^{2} / \mathrm{s}\right.$ at $\left.18^{\circ} \mathrm{C}\right)$ and finally vinyl flooring with the lowest $\left(9.17 \times 10^{-14} \mathrm{~m}^{2} / \mathrm{s}\right.$ at $\left.18^{\circ} \mathrm{C}\right)$. These results not only show diffusion of a gaseous species through a porous media but support the case for $\mathrm{CO}$ diffusion as $\mathrm{CO}$ has a smaller molecule size than formaldehyde and therefore it can diffuse more easily.

Diffusion through a gypsum board was found in Blondeau et al. (2003). It aimed to determine the diffusion of ethyl acetate $\left(\mathrm{CH}_{3}-\mathrm{COO}-\mathrm{CH}_{2}-\mathrm{CH}_{3}\right)$ and n-octane $\left(\mathrm{C}_{8} \mathrm{H}_{18}\right)$ in building materials. The computed effective diffusivities for various building materials were subsequently compared to data from previous experiments, showing good agreement. The calculated effective diffusivity of ethyl acetate and n-octane through gypsum board are around $1.2 \times 10^{-6} \mathrm{~m}^{2} / \mathrm{s}$ for the former and $0.9 \times 10^{-6}$ $\mathrm{m}^{2} / \mathrm{s}$ for the latter. It should be noted that in this experiment these gases both have larger molecules than $\mathrm{CO}$ and hence, under the same conditions, one would assume that $\mathrm{CO}$ would diffuse to a greater extent if not to a similar extent.

Further examples of diffusion of ethyl acetate and n-octane through gypsum wallboard were shown in Meininghaus et al. (2000). The purpose was to present quantitative experimental results on diffusion and sorption of volatile organic compounds (VOC) in indoor materials and was done using a Climpaq style chamber (Gunnarsen et al., 1994), the edges of which were sealed to inhibit air leakage. It was found that mass transport of these gases can occur very quickly, with some effective diffusion coefficients being one order of magnitude below those found in air-similar to the findings in Blondeau et al. (2003). Also, it was found that gypsum board showed the highest diffusion coefficient of all studied materials.

Further diffusion through building materials was found reported in Meininghaus and Uhde (2002). In this paper the mass flow rate of VOC mixtures across a gypsum board was studied using two setups, both of which include a FLEC (Field and Laboratory Emission Cell) and were sealed with either Teflon or aluminum tape to ensure no air leakage. The results of this paper showed that the transport of certain VOC across a gypsum board could be fast. Furthermore, it was found that the mass transport was dependent on molecular properties such as the boiling point and the molecular area and that similar compounds show similar mass transport processes.

Hence, from these cases we can see that the support for carbon monoxide diffusing through gypsum is well documented. The experimental observation of gases with increased molecular mass diffusing through gypsum wallboard alludes to the possibility of carbon monoxide diffusing through gypsum wallboard, as the ability of a molecule to undergo diffusion increases with decreasing molecular mass.

\section{Reported Incidents}

It was found that most reported cases of $\mathrm{CO}$ intoxication in the literature were attributed to vehicles and appliances in the same room as the victim of the intoxication with little details being given about the cases involving a potential $\mathrm{CO}$ source located in a non-communicating area. This is due to the lack of understanding of whether CO can transport through non-communicating rooms. However, there are a few available reported incidents that deal with potential instances where $\mathrm{CO}$ transport took place through walls.

Three incidents are highlighted (Keshishian et al., 2012) where $\mathrm{CO}$, produced in neighboring restaurants, traveled through the walls and floor and resulted in toxic levels within the adjacent residencies. All three restaurants used charcoal-burning ovens or grills which, although ventilated during the day, were left smoldering overnight with the ventilation turned off, resulting in a build-up of CO. These periodic accumulations of $\mathrm{CO}$ were seen reflected in the residencies indicating that the levels in the two properties were not independent of one another and that transport of the gas was taking place. Because there were no communicating openings between the restaurants and the homes, it was concluded that the most probable scenario was that CO traveled through diffusion.

A similar situation was reported in West (2008) in which a neighboring restaurant was influencing $\mathrm{CO}$ levels within a residency. The report focuses on identifying the symptoms of $\mathrm{CO}$ poisoning and on giving recommendations on the optimal ventilation to avoid build-up of CO. While the restaurant was placed below the apartment, there are no further descriptions of the configuration. However, it is most probable that the transport of $\mathrm{CO}$ was through the floor.

An incident was reported by OSHA (2012) in which the exhaust of a swimming pool natural-gas heater was channeled through a detached pipe through four of the five floors of a hotel building, contained within a large shaft. However, the ventilation 
system was not functioning correctly and a build-up of CO was produced within the shaft. As a consequence two employees, in a room adjacent but not communicating to the shaft, were affected by CO poisoning.

It was reported in Hampson (2009) that levels of CO in a first floor bedroom were being affected by emission from a water heater in the ground floor utility room. However, this report is just a reply to an article, therefore the incident is not detailed extensively and so one can only assume that diffusion or air leakage was the main transport mechanism.

Many reports of $\mathrm{CO}$ intoxication focus mostly on presenting the symptoms of $\mathrm{CO}$ poisoning and identifying the source which produces $\mathrm{CO}$, as well as recommendations for avoiding intoxication. Details such as building materials, presence of vents or openings are not presented, making it difficult to pinpoint diffusion as a means of transport of $\mathrm{CO}$ through walls. However, the cases presented support the evidence of CO transport through walls, given that there were no clear communicating openings between the $\mathrm{CO}$-producing room and the adjacent rooms where high levels of $\mathrm{CO}$ were measured.

\section{METHOD}

The experiments that signaled the possibility of diffusion of CO through gypsum wallboards (Hampson, Courtney, \& Holm, Diffusion of Carbon Monoxide Through Gypsum Wallboard, 2013) were carried out in a test chamber made of Plexiglass supported by a wooden frame, with the exterior dimensions of 0.6 by 0.6 by $2.44 \mathrm{~m}$ ( 24 by 24 by 96 inches) and sealed with silicone caulk at all junctions, as shown in Figure 1. The chamber consisted of two sides separated by a gypsum wallboard of various thicknesses (single layer $0.25^{\prime \prime}-6.35 \mathrm{~mm}$ - and $0.5^{\prime \prime}-12.7$ $\mathrm{mm}$-, as well as double layer $0.5^{\prime \prime}$ and double layer $0.5^{\prime \prime}$ painted). Carbon monoxide test gas at 3,000 ppm was infused on one side at $15 \mathrm{l} / \mathrm{min}$ until it reached a concentration of 500-600 ppm. Measurements were taken at the control side every $1 \mathrm{~min}$ for $24 \mathrm{~h}$, in order to establish how long it takes for the concentration to reach levels that affects humans (100 ppm). Figure 2 shows a summary of these experiments, presenting on the left side the $\mathrm{CO}$ concentration levels in the chamber where the gas was infused for every configuration used. The right side of the figure shows the $\mathrm{CO}$ concentration levels in the control chamber after $\mathrm{CO}$ diffused through the wall. The complete raw data set can be found online (Hampson, 2014). It was found that depending on the configuration, this concentration was reached in 17-96 min. Also, the $\mathrm{CO}$ concentration in both chambers differed by only $5 \%$ after $12 \mathrm{~h}$.

Although the experimental procedure by Hampson (2014) could be improved to be more detailed on the conditions and diagnostics, the experimental results provided by Hampson (2014) are sufficient to produce a computational model to replicate and verify that the findings agree with fundamental mass diffusion theory and that $\mathrm{CO}$ can in fact diffuse through gypsum walls. This model is a simplified 1D mass transfer model which assumes well-mixed CO in the chamber, an assumption which is investigated further on. The equations for this model, as well as the initial conditions are specified in Equation (1-5), where $c_{1}$ and $c_{2}$ are the concentration in the infusion (first) and control (seconds) chamber, which are dependent on time, $c_{1^{0}}$ is the initial $\mathrm{CO}$ concentration in the infusion chamber, and $\mathrm{K}$ is a constant. $\mathrm{K}\left(\mathrm{s}^{-1}\right)$ is a diffusion parameter that is found by equating the mass lost from a chamber per unit time to the flux by diffusion. It is represented by Equation (6), where D is the diffusivity of the gas into the gypsum board, units of $\mathrm{m}^{2} \mathrm{~s}^{-1}, \mathrm{~A}$ $\left(\mathrm{m}^{2}\right)$ the area of the gypsum board, L $(\mathrm{m})$ the thickness of the gypsum board, $\mathrm{V}\left(\mathrm{m}^{3}\right)$ is the volume of the tank.

$$
\begin{aligned}
& \frac{d c_{1}}{d t}=\left(c_{2}-c_{1}\right) K \\
& \frac{d c_{2}}{d t}=\left(c_{1}-c_{2}\right) K
\end{aligned}
$$

Initial conditions:

$$
\begin{array}{r}
c_{1}(0)=c_{1}^{0} \\
c_{2}(0)=0 \\
c_{1}(t)=\frac{c_{1}^{0}}{2}\left(1+e^{-2 K t}\right) \\
c_{2}(t)=\frac{c_{1}^{0}}{2}\left(1-e^{-2 K t}\right) \\
K=\frac{D A}{L V}
\end{array}
$$

When calculating the mean diffusivity, we ignore the transient and study the values calculated from measurements in the nontransient diffusion region, i.e. after the mixing of the gas in the infusion side of the tank is complete. The experimental setup shown in Figure $\mathbf{1}$ is at ambient temperature and pressure, $\mathrm{CO}$ is a gas at all times and does not form precipitates or aerosols which otherwise would affect the validity of Equations $(1,2)$. The corners of the chambers and the wall pores might create small stagnation volumes, which are not taken into account by the $1 \mathrm{D}$ mass transfer model due to the well-mixed $\mathrm{CO}$ assumption. However, this combined volume is negligible.

Mass conservation was invoked in the analysis, but in the experimental data there were mass losses in the system. Mass losses were probably due to leakage out of the tanks. Mass absorption could be a minimal component of the mass loss, but as the ratio of surface over which mass adsorption could potentially take place in respect to the total volume of the tank is minimal this contribution is very small. To quantify these, mass loss out of the setup during the first $10 \mathrm{~h}$ of the experiment was also calculated. This was done by summing $c_{1}(t)+c_{2}(t)$ and comparing it to $c_{1}^{0}$. Ten hour was chosen as the length of time to ensure that in all the different experiments the concentration on both sides of the setup had stabilized, so as to ensure that all relevant diffusive processes are included in this analysis. Mass loss is less than $8 \%$ for the published experiments $\left(0.5^{\prime \prime}\right.$ gypsum wallboard), but is considerably greater for those at the other thicknesses $\left(0.25^{\prime \prime}\right.$, double $0.5^{\prime \prime}$, double $0.5^{\prime \prime}$ painted). Therefore mass was not conserved in all experiments, which is another factor not considered in the model proposed here since the equations assume mass conservation. The mass loss can be explained by the $\mathrm{CO}$ being absorbed by the Plexiglass walls or leaking through the junctions. However, the ratio of surface over 
which mass adsorption could potentially take place in respect to the total volume of the tank is minimal, so the contribution of mass adsorption to the mass loss is small, and the likely reason for the mass loss is leakages through the junctions.

The initial value of $\mathrm{CO}$ in the infusion was taken to be the value of $\mathrm{CO}$ present after the mixing was complete. Therefore

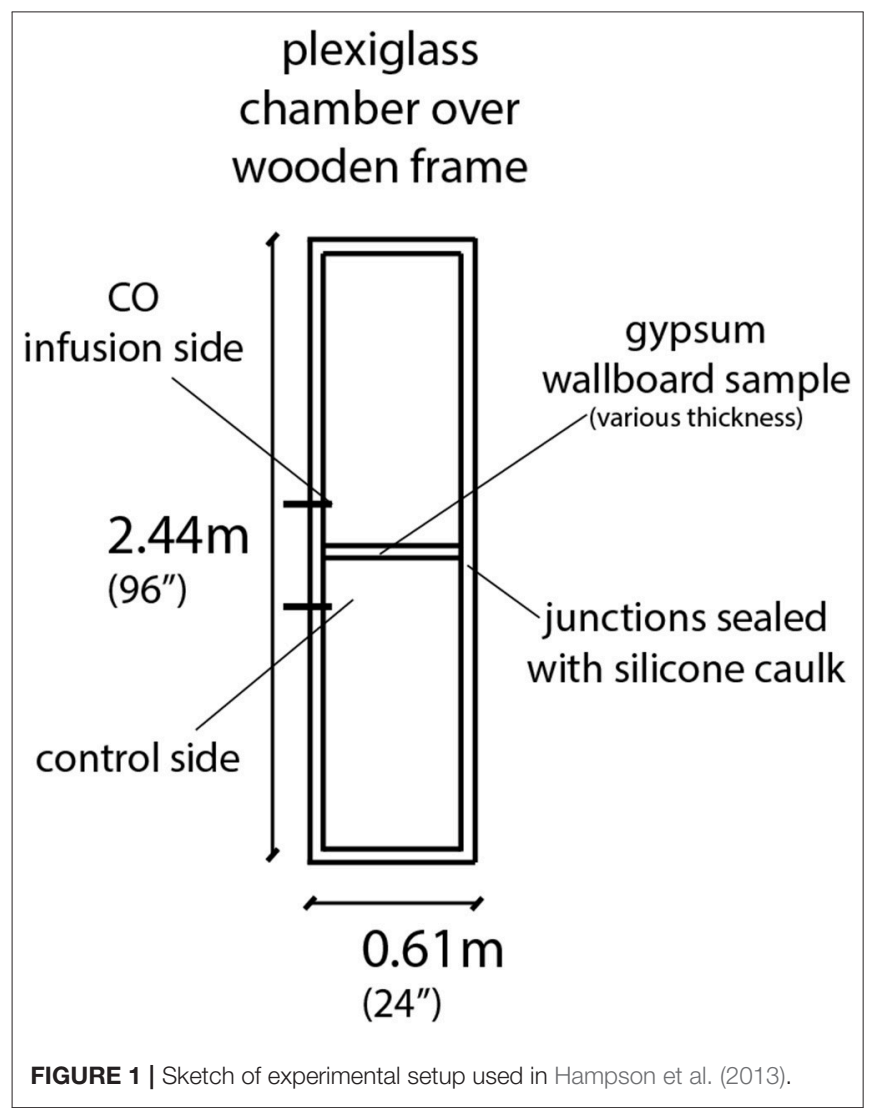

we ignore the mixing time and assume perfectly mixed gases in the diffusion tank for the model. In the experimental data, the initial CO concentration reported is different because it is measured in the mixing period prior to the well-mixed state being reached. Note that the effective diffusivity $D_{e}$ is calculated using the values from Eq. 7 and 8, and averaging the two as shown in Equation (9). This means that diffusivity is being calculated with concentrations from both sides of the tank.

$$
\begin{array}{r}
D_{1}=\frac{-V L}{2 A t} \ln \left(\frac{2 c_{1}}{c_{1}^{0}}-1\right) \\
D_{2}=\frac{-V L}{2 A t} \ln \left(1-\frac{2 c_{2}}{c_{1}^{0}}\right) \\
D_{e}=\frac{D_{1}+D_{2}}{2}
\end{array}
$$

The effective diffusivity $D_{e}$ is calculated averaging the $\mathrm{D}$ values provided by Equations $(7,8)$ onwards $15 \mathrm{~min}$ since $\mathrm{CO}$ infusion (to assume well-mixed conditions) until the infusion side of the tank reaches a $\mathrm{CO}$ concentration half its initial value, which is considered to be the theoretical steady-state point.

\section{RESULTS}

The ranges of diffusivities found are between $1.6 \times 10^{-6}$ and $4.0 \times 10^{-6} \mathrm{~m}^{2} \mathrm{~s}^{-1}$. The difference in values can be explained by differences in mass losses for each experiment and slight variation in the experimental setup when changing the thicknesses of the gypsum board, but all of the results are in the same order of magnitude. The results for the mean diffusivities are gathered in Table 1.

The test case chosen to be presented fully is test 2 from the $0.5^{\prime \prime}$ gypsum wallboard, which was also shown in Hampson et al. (2013). Figure 3 shows the mass loss over $10 \mathrm{~h}$, where the dotted line represents the initial CO concentration in the infusion
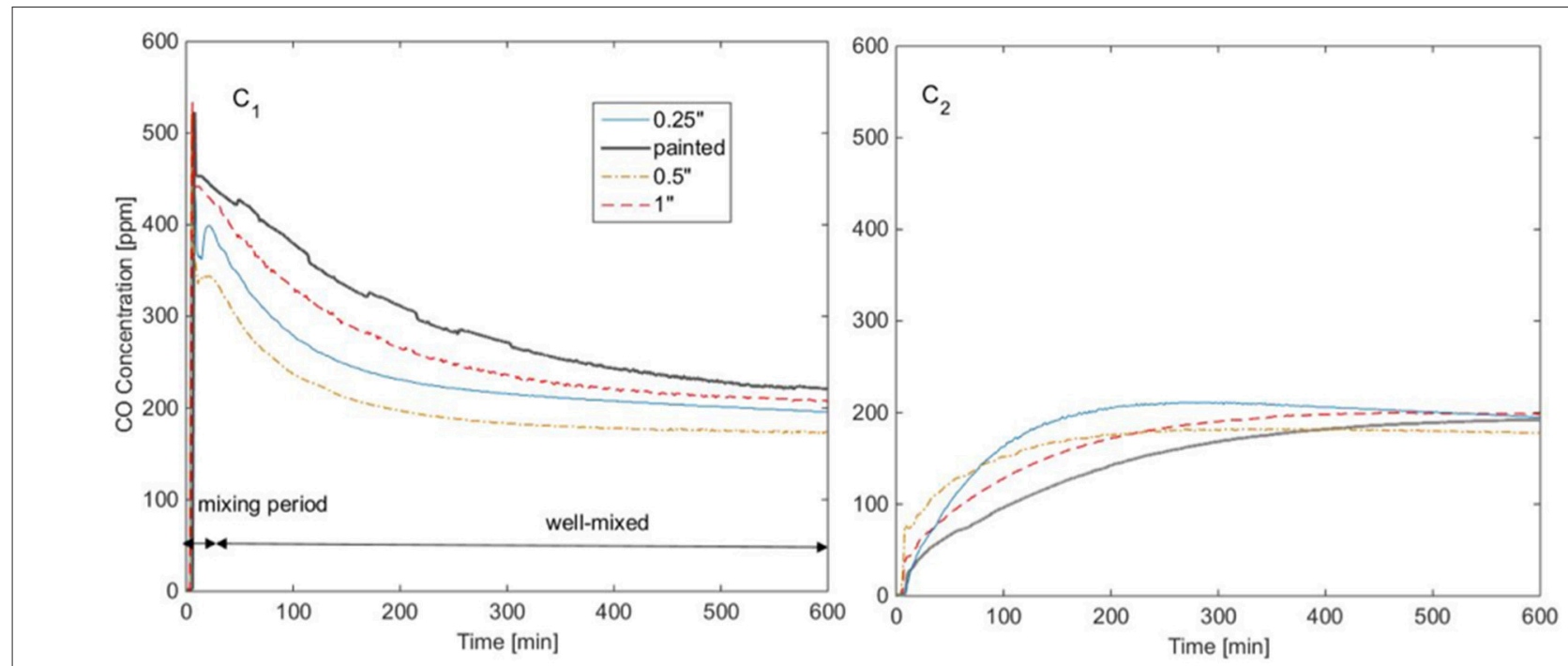

FIGURE 2 | Summary of the experiments in Hampson et al. (2013); left: CO concentration in the infusion chamber, right: CO concentration in the control chamber. 
TABLE 1 | The values of calculated effective diffusivities for all 12 experiments.

\begin{tabular}{|c|c|c|c|}
\hline Test & $c_{1}^{0}$ & $\begin{array}{l}\text { Mean D } \\
{\left[\mathrm{m}^{2} \mathrm{~s}^{-1}\right]}\end{array}$ & $\begin{array}{c}\% \text { Mass loss } \\
\text { after } \\
10 \mathrm{~h}\left(\mathrm{~m}_{l}\right)(\%)\end{array}$ \\
\hline $0.25^{\prime \prime}$ - Test 1 & 480 & $1.71 \times 10^{-6}$ & 14.2 \\
\hline $0.25^{\prime \prime}$ - Test 2 & 440 & $1.75 \times 10^{-6}$ & 11.1 \\
\hline $0.25^{\prime \prime}$ - Test 3 & 470 & $1.60 \times 10^{-6}$ & 10.2 \\
\hline $0.5^{\prime \prime}$ - Test 1 & 330 & $4.03 \times 10^{-6}$ & 6.1 \\
\hline $0.5^{\prime \prime}$ - Test 2 & 380 & $4.00 \times 10^{-6}$ & 7.4 \\
\hline $0.5^{\prime \prime}$ - Test 3 & 350 & $4.06 \times 10^{-6}$ & 4.9 \\
\hline $1^{\prime \prime}-$ Test 1 & 490 & $4.80 \times 10^{-6}$ & 18.0 \\
\hline $1^{\prime \prime}$ - Test 2 & 490 & $5.00 \times 10^{-6}$ & 17.6 \\
\hline 1" - Test 3 & 485 & $4.46 \times 10^{-6}$ & 15.8 \\
\hline Painted $1 "$ - Test 1 & 500 & $2.89 \times 10^{-6}$ & 15.6 \\
\hline Painted $1^{\prime \prime}$ - Test 2 & 485 & $3.08 \times 10^{-6}$ & 14.8 \\
\hline Painted $1^{\prime \prime}$ - Test 3 & 495 & $3.19 \times 10^{-6}$ & 14.1 \\
\hline
\end{tabular}

chamber $c_{1^{0}}$ and the solid line the sum of the concentrations on both sides of the setup $c_{1}(t)+c_{2}(t)$. This shows that there are some leaks in the overall experimental system, despite the sealed junctions. Figure 4 shows the calculated diffusivity, having an average of $4 \times 10^{-6} \mathrm{~m}^{2} \mathrm{~s}^{-1}$. Figure 5 directly compares the results from the experiments with the model results which use the calculated diffusivities. They are in the same order of magnitude with the results of separate tests carried out by Cleary et al. (2014). The values of the effective diffusivities are summarized in Figure 6 where they are compared to Cleary's test results as well as the effective diffusivity of several other gases through gypsum found in literature and given for reference (Blondeau et al., 2003). It should be noted however, that these values do not represent the diffusion, which is clearly faster for thinner materials, but the diffusivity which is a material property and thus does not depend on thickness. The differences in diffusivities stem from the errors from the experimental setup as explained in the previous paragraph, but all of the results are within the same order of magnitude. As all the experiments carried some mass loss, we found the range of resulting average diffusivities by calculating the average diffusivity with zero mass loss incorporated in the model, and maximum mass loss. We use the values of \% mass loss after $10 \mathrm{~h}$ reported in Table 1 to carry out a sensitivity analysis of the effective diffusivity in our $1 \mathrm{D}$ model in respect to mass loss seen in the experiment. These results are shown in Table 2, and for all cases they are in the same order of magnitude as the original model, with a maximum error of $13.75 \%$ for all the unpainted gypsum board tests, and a high error for a single painted board test. All diffusivities found in this sensitivity analysis were of the same order of magnitude showing that the $1 \mathrm{D}$ model does not present a large dependency on leaks.

\section{DISCUSSION}

The objective of this study was to verify the experiments in Hampson et al. (2013) through a mass diffusion model and to investigate incident reports and literature papers that

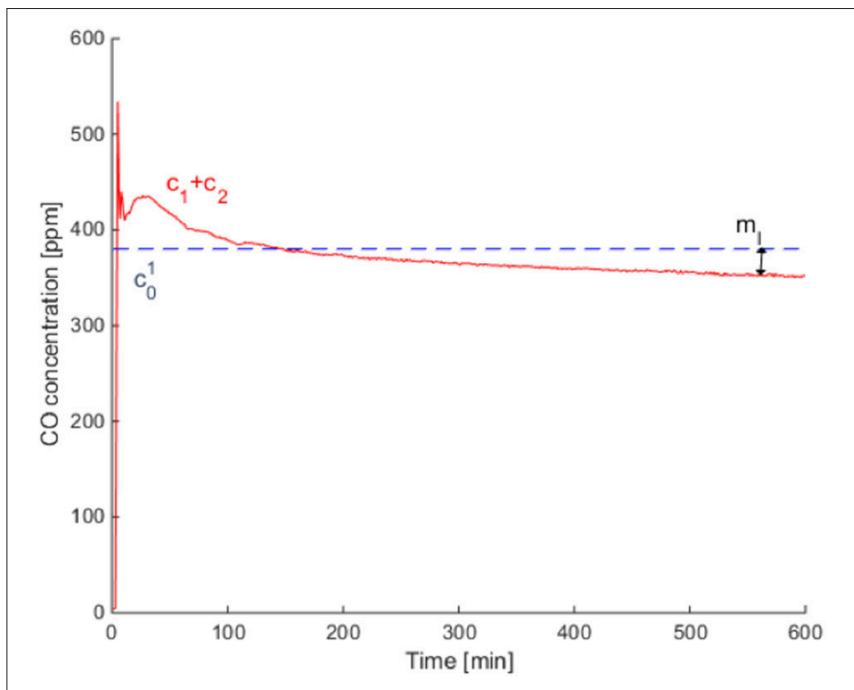

FIGURE $\mathbf{3}$ | Mass loss rate comparison for $0.5^{\prime \prime}$ test 2. $\mathbf{c}_{\mathbf{1}} \mathbf{0}$ is compared to $\mathbf{c}_{\mathbf{1}}(\mathbf{t})+\mathbf{c}_{\mathbf{2}}(\mathbf{t})$ over $10 \mathrm{~h}$.

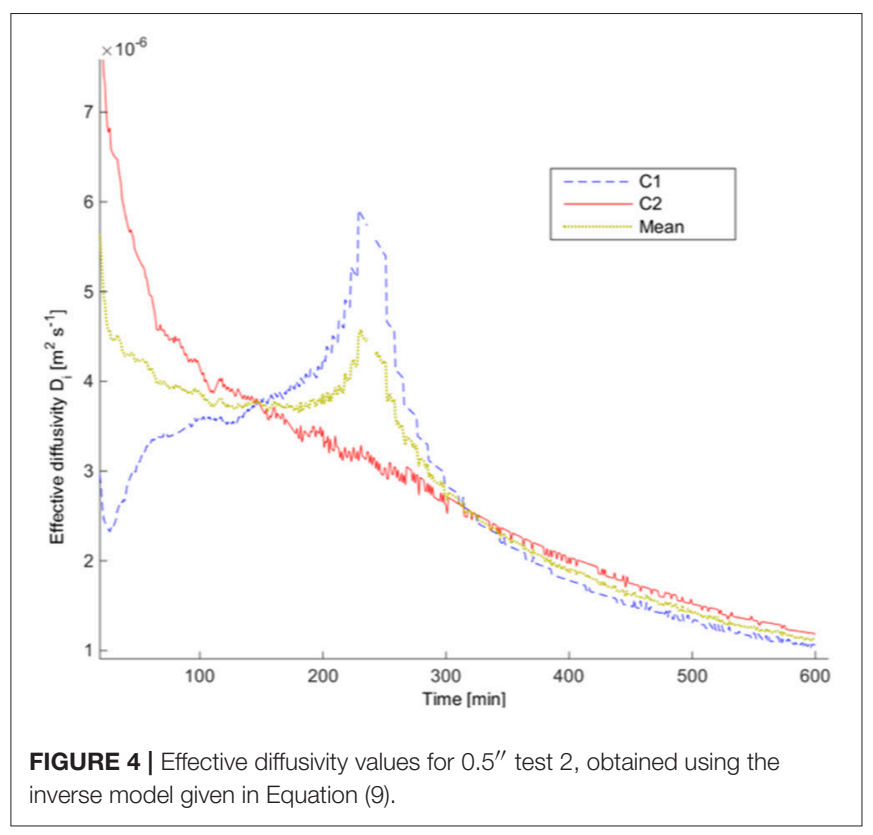

provide examples of $\mathrm{CO}$ or carbohydrates diffusing through porous media. The simple mass transfer model confirms the fast transport of $\mathrm{CO}$ through porous walls, independently assessing the experimental results from Hampson et al. (2013). This is further demonstrated by obtaining results in the same order of magnitude as another parallel computational study by Cleary et al. (2014). However, experimental uncertainty should be kept in mind, especially at the junctions which were sealed with silicone caulk. The computational model and previously published experimental results show good agreement, and complementing these with literature studies of $\mathrm{CO}$ and carbohydrates diffusing through porous media 
highlights the danger posed by $\mathrm{CO}$ in rooms adjacent to enclosures containing $\mathrm{CO}$ sources such as garages or kitchens.

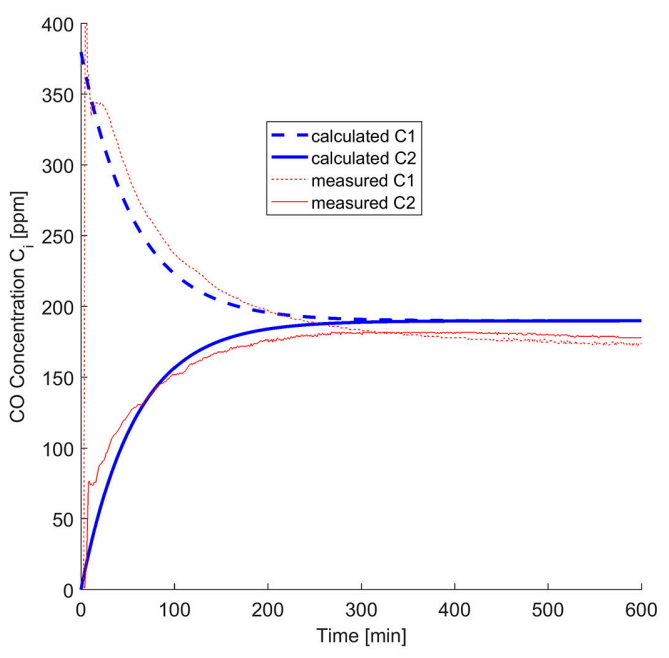

FIGURE 5 | Comparison between the experimental results and the calculated diffusivity for experimental data using $0.5^{\prime \prime}$ gypsum wallboard.

\section{CONCLUSION}

The literature review offers support to the claim that carbon monoxide can diffuse through porous walls at a rate that presents a danger to the occupants. There are experiments in literature that use various VOC that prove gases are able to migrate through

TABLE 2 | Sensitivity analysis of calculated effective diffusivities for all 12 experiments using maximum mass losses.

\begin{tabular}{|c|c|c|c|}
\hline Test & $\begin{array}{c}\text { Mean D } \\
{\left[\mathrm{m}^{2} \mathrm{~s}^{-1}\right]}\end{array}$ & $\begin{array}{c}\text { Mean } D \text { at } \\
\text { maximum mass } \\
\text { loss }\left[\mathrm{m}^{2} \mathrm{~s}^{-1}\right]\end{array}$ & $\begin{array}{l}\text { Percentage } \\
\text { difference }\end{array}$ \\
\hline $0.25^{\prime \prime}$ - Test 1 & $1.71 \times 10^{-6}$ & $1.65 \times 10^{-6}$ & 3.51 \\
\hline $0.25^{\prime \prime}$ - Test 2 & $1.75 \times 10^{-6}$ & $1.78 \times 10^{-6}$ & 1.12 \\
\hline $0.25^{\prime \prime}$ - Test 3 & $1.60 \times 10^{-6}$ & $1.82 \times 10^{-6}$ & 13.75 \\
\hline $0.5^{\prime \prime}$ - Test 1 & $4.03 \times 10^{-6}$ & $3.98 \times 10^{-6}$ & 1.24 \\
\hline $0.5^{\prime \prime}$ - Test 2 & $4.00 \times 10^{-6}$ & $3.80 \times 10^{-6}$ & 5.00 \\
\hline $0.5^{\prime \prime}$ - Test 3 & $4.06 \times 10^{-6}$ & $3.83 \times 10^{-6}$ & 5.67 \\
\hline $1^{\prime \prime}$ - Test 1 & $4.80 \times 10^{-6}$ & $4.30 \times 10^{-6}$ & 10.42 \\
\hline $1^{\prime \prime}$ - Test 2 & $5.00 \times 10^{-6}$ & $5.30 \times 10^{-6}$ & 6.00 \\
\hline $1^{\prime \prime}-$ Test 3 & $4.46 \times 10^{-6}$ & $4.20 \times 10^{-6}$ & 5.83 \\
\hline Painted $1 "$ - Test 1 & $2.89 \times 10^{-6}$ & $3.66 \times 10^{-6}$ & 26.64 \\
\hline Painted 1" - Test 2 & $3.08 \times 10^{-6}$ & $3.80 \times 10^{-6}$ & 42.11 \\
\hline Painted $1 "$ - Test 3 & $3.19 \times 10^{-6}$ & $3.79 \times 10^{-6}$ & 18.50 \\
\hline
\end{tabular}

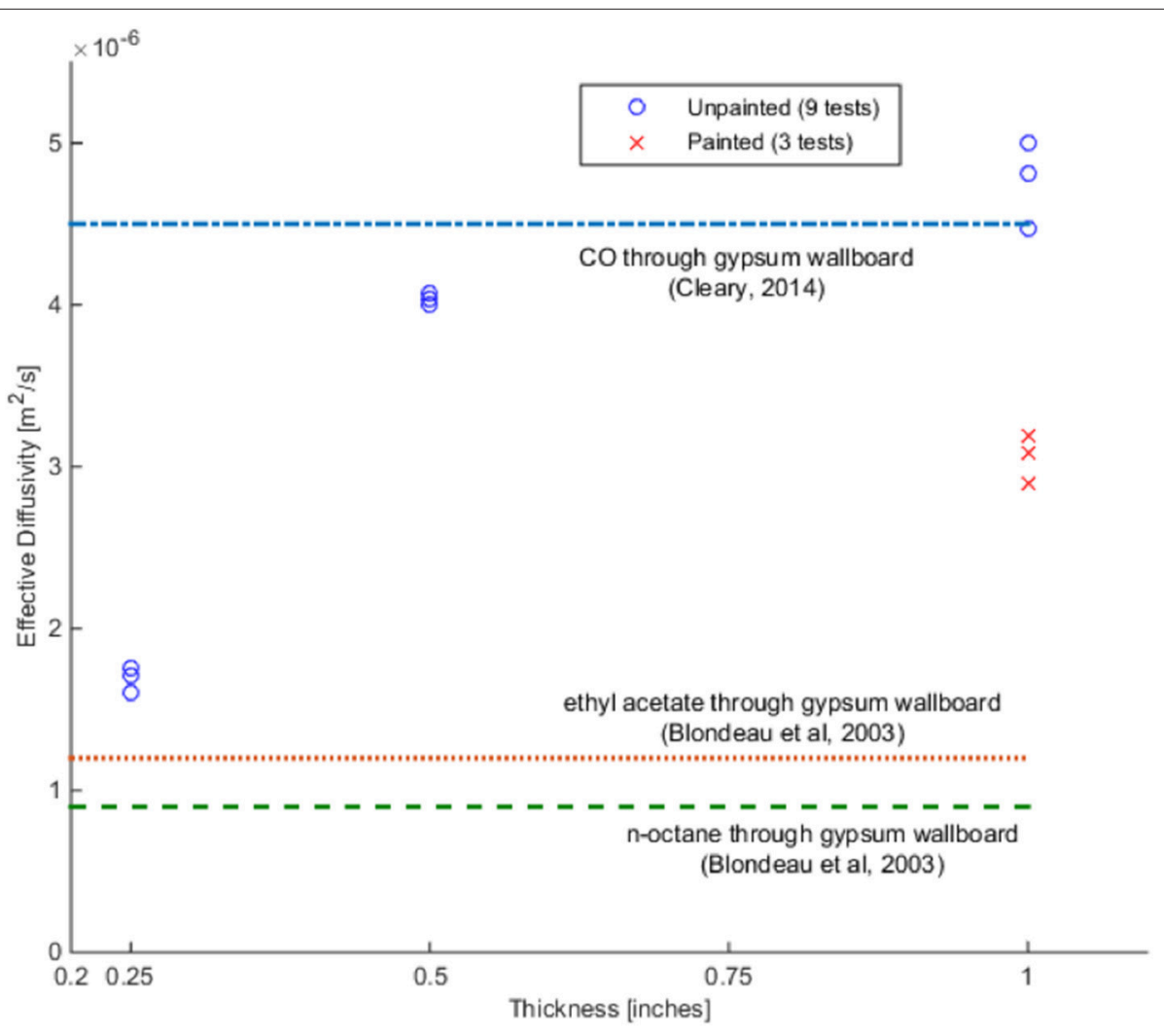

FIGURE 6 | Comparison of mean diffusivities for the 12 experiments, along with values from literature. 
the pores in the walls. As CO is a smaller molecule than these, it can be concluded that it can diffuse at least as fast as those. In addition, experiments that replicate realistic conditions have shown the ability of CO to diffuse through walls.

There have been 5 reported incidents of carbon monoxide intoxication which can be attributed to diffusion, with one additional incident where diffusion is thought to have contributed to the high concentration of CO in two separate rooms. These reports do not give many details about the building materials and give basic information about the configuration, but from what they provide it is very likely that $\mathrm{CO}$ can diffuse through walls. Therefore, there is a need to conduct an investigation into the possibility of $\mathrm{CO}$ diffusing through porous walls.

The mass transfer model made to verify the experimental results of Hampson et al. (2013) that have brought attention to this phenomenon gave conclusive results. Although there could have been more diagnostics and details in the experimental procedure by Hampson (2014) there is sufficient information to produce the computational model to verify that $\mathrm{CO}$ can in fact diffuse through gypsum walls, a phenomenon not taken into consideration until now. The diffusivity of $\mathrm{CO}$ across gypsum board can be quantified and it is inside the range from $1.6 \times 10^{-6}$ to $4 \times 10^{-6} \mathrm{~m}^{2} / \mathrm{s}^{-1}$. This range is in the same order of magnitude as the results obtained recently for the same materials by researchers at NIST (Cleary et al., 2014).

\section{REFERENCES}

Blondeau, P., Tiffonnet, A. L., Damin, A., Amiri, O., and Molina, J. L. (2003). Assessment of contaminant diffusivities in building materials from porosimetry tests. Indoor Air 13, 302-310. doi: 10.1034/j.1600-0668.2003. 00208.x

Chang, J., and Guo, Z. (1992). The effects of building features on air and pollutant movement. Build. Res. J. 1, 55-63.

Cleary, T., Yang, J., and Fernandez, M. (2014). New Research on Diffusion of Carbon Monoxide through Gypsum Wallboard. Gaithersburg, MD.

Deng, Q., Yang, X., and Zhang, J. (2009). Study on a new correlation between diffusion coefficient and temperature in porous building materials. Atmos. Environ. 43, 2080-2083. doi: 10.1016/j.atmosenv.2008.12.052

Gilliland, E., Baddour, R., Perkinson, G., and Sladek, K. (1974). Diffusion on surfaces. 1. effect of concentration on the diffusivity of physically. Indus. Eng. Chem. Fundamentals 13, 95-100. doi: 10.1021/i160050a001

Gunnarsen, L., Nielsen, P., and Wolkoff, P. (1994). Design and characterization of the CLIMPAQ, chamber for laboratory investigations of materials, pollution and air quality. Indoor Air 4, 56-62.

Hampson, N. (2009). Saved by the Carbon Monoxide. Am. J. Prevent. Med. 37:473. doi: 10.1016/j.amepre.2009.08.015

Hampson, N. (2014). CO Bibliography. [Online] Available Online at: http:// courtney.neilhampson.com/carbon-monoxide.html (Accessed December 2014).

Hampson, N. B., Courtney, T. G., and Holm, J. R. (2013). Diffusion of carbon monoxide through gypsum wallboard. JAMA 310, 745-746. doi: 10.1001/jama.2013.43127

Incropera, F., Dewitt, D., Bergman, T., and Lavine, A. (2013). Principles of Heat and Mass Transfer, 7th Edn. Hoboken, NJ John Wiley and Sons.

Keshishian, C., Sandle, H., Meltzer, M., Young, Y., Ward, R., and Balasegaram, S. (2012). Carbon monoxide from neighbouring restaurants: the need for an integrated multi-agency response. J. Public Health 34, 477-482. doi: 10.1093/pubmed/fds023
Along with the paper by Hampson et al. (2013), this work provides an initial answer to the question of the possibility of $\mathrm{CO}$ diffusing through a porous wall. With the simple mass transfer model independently confirming the correctness of the experiments, this represents an initial step in the investigation of $\mathrm{CO}$ diffusion through porous walls. Future work should aim to produce an in-depth experimental and computational investigation into the specifics of $\mathrm{CO}$ diffusion through porous walls.

\section{AUTHOR CONTRIBUTIONS}

GR was the principal investigator of this project. CW-R did the initial literature review. FR worked on the mathematical model. IV put the paper together.

\section{ACKNOWLEDGMENTS}

This research has been funded by the Fire Protection Research Foundation (FPRF) and is available online as the original FPRF report in February 2015. The authors thank the help of the FPRF Advisory Panel chaired by Amanda Kimball. The authors would also like to thank Dr. Neil Hampson (Virginia Mason Medical Center) for providing his experimental data. The authors wish to express their gratitude to the Imperial College Trust Fund and the Old Centralians' Trust for their financial support.

Kontogeorgos, D., and Founti, M. (2013). A generalized methodology for the definition of reactive porous materials physical properties: prediction of gypsum board properties. Constr. Build. Mater. 48, 804-813. doi: 10.1016/j.conbuildmat.2013.07.095

Meininghaus, R., Gunnarsen, L., and Knudsen, H. (2000). Diffusion and sorption of volatile organic compounds in building materials-impact on indoor air quality. Environ. Sci. Tech. 34, 3101-3108. doi: 10.1021/es991291i

Meininghaus, R., and Uhde, E. (2002). Diffusion studies of VOC mixtures in building materials. Indoor Air. 2, 215-222. doi: 10.1034/j.1600-0668.2002.01131.x

Nelson, G. (1998). Carbon monoxide and fire toxicity: a review and analysis of recent work. Fire Technol. 34, 39-58. doi: 10.1023/A:1015308915032

NFPA (2012). NFPA 720: Standard for the Installation of Carbon Monoxide (co) Detection and Warning Equipment. NFPA

NFPA (2015). NFPA 101: Life Safety Code. NFPA

OSHA (2012). Accident Investigation Summary, South Charleston WV: OSHA.

Singer, B., Revzan, K. L., Hotchi, T., Hodgson, A. T., and Brown, N. J. (2004). Sorption of organic gases in a furnished room. Atmos. Environ. 38, 2483-2494. doi: 10.1016/j.atmosenv.2004.02.003

Treybal, R. E. (1981). Mass-Transfer Operations. Paris: McGraw-Hill International. West, F. (2008). Odour as a Potential Proxy for Carbon Monoxide Exposure.

Conflict of Interest Statement: The authors declare that the research was conducted in the absence of any commercial or financial relationships that could be construed as a potential conflict of interest.

Copyright (c) 2018 Vermesi, Restuccia, Walker-Ravena and Rein. This is an openaccess article distributed under the terms of the Creative Commons Attribution License (CC BY). The use, distribution or reproduction in other forums is permitted, provided the original author(s) and the copyright owner(s) are credited and that the original publication in this journal is cited, in accordance with accepted academic practice. No use, distribution or reproduction is permitted which does not comply with these terms. 